


\title{
Orbital exenterations and squamous cell carcinoma of the conjunctiva at Sekuru Kaguvi Eye Unit, Zimbabwe
}

\author{
*R MASANGANISE, **A MAGAVA
}

\begin{abstract}
Objectives: The aim of the audit was to determine the common orbital diseases necessitating orbital exenterations at Sekuru Kaguvi Hospital (SKH) between January and December 1999 and recommend ways of reducing the numbers of procedures done annually.

Design: Retrospective cross sectional study.

Setting: Department of Surgery, Parirenyatwa Hospital, University of Zimbabwe.

Subjects: 23 patients who underwent orbital exenterations at SKH over a one year period.

Main Outcome Measure: The nature of orbital tumours requiring orbital exenteration in Zimbabwe.

Results: A total of 23 patients underwent orbital exenteration during the period under review. Of the 23 patients, $13(56.5 \%$ ) had squamous cell carcinoma (SCCA) of the conjunctiva with orbital extensions, $69 \%$ of the 13 patients were females and the mean age of these patients was 37.2 years (interquartile range 28,48 ). Conclusions: People in tropical regions, where there is a high risk of squamous cell carcinoma of the conjunctiva in terms of increased predisposition to solar radiation, high prevalence of HIV and inadequate eye care services, should seek medical attention as soon as they notice a persistent conjunctival growth. Conjunctival tumours should be widely excised and material sent for histology to confirm diagnosis and clarity of excision margins. All patients diagnosed as having SCCA of the conjunctiva should be closely monitored to facilitate early detection of recurrences and institute appropriate treatment.
\end{abstract}

Cent Afr J Med 2001;47(8):196-9

\author{
*Department of Surgery \\ University of Zimbabwe Medical School \\ PO Box A178, Avondale \\ Harare, Zimbabwe \\ **:Sekuru Kaguvi Eye Unit \\ Parirenyatwa Hospital \\ Harare, Zimbabwe
}

Correspondence to:

Dr Rangarirai Masanganise

Tel 263 (4) 791631 ext 2169

Fax: 263 (4) 791995 or 724912

E-mail:ceu@healthnet.zw

Cent Afr J Med 2001;47(8) 


\section{Introduction}

The association of squamous cell carcinomas of the conjunctiva and human immunodeficiency virus (HIV) is well documented in the literature. ${ }^{1-3}$ Early detection and treatment of patients with SCCA of the conjunctiva is of utmost importance in terms of curing the disease and minimising the problems associated with managing advanced disease. Inadequate ophthalmic services in Zimbabwe coupled with harsh socio-economic circumstances contribute to late presentation of patients with SCCA of the conjunctiva, thus necessitating the use of orbital exenteration as the most appropriate management option.

Orbital exenteration is a surgical procedure involving the removal of all orbital contents including eyelids up to the brow leaving the bony walls. The procedure is usually recommended for patients with advanced orbital malignancies. It facilitates the delivery of both radiotherapy and chemotherapy, ${ }^{4}$ offers the patient a chance of complete recovery from the disease and also improves the quality'of life for the patient. The procedure is very rare among developed countries and was fairly uncommon in our department during the eighties. Anecdotal reports suggest that the trend of orbital exenterations at Sekuru Kaguvi Eye Unit ( $\mathrm{SKH}$ ) has changed during the last decade. However, the procedure is not without problems namely: cosmetic (Figure I), slow healing, risk of bone necrosis and infection, recurrence of the disease and the complex

Figure I: Patient who had orbital exenteration.

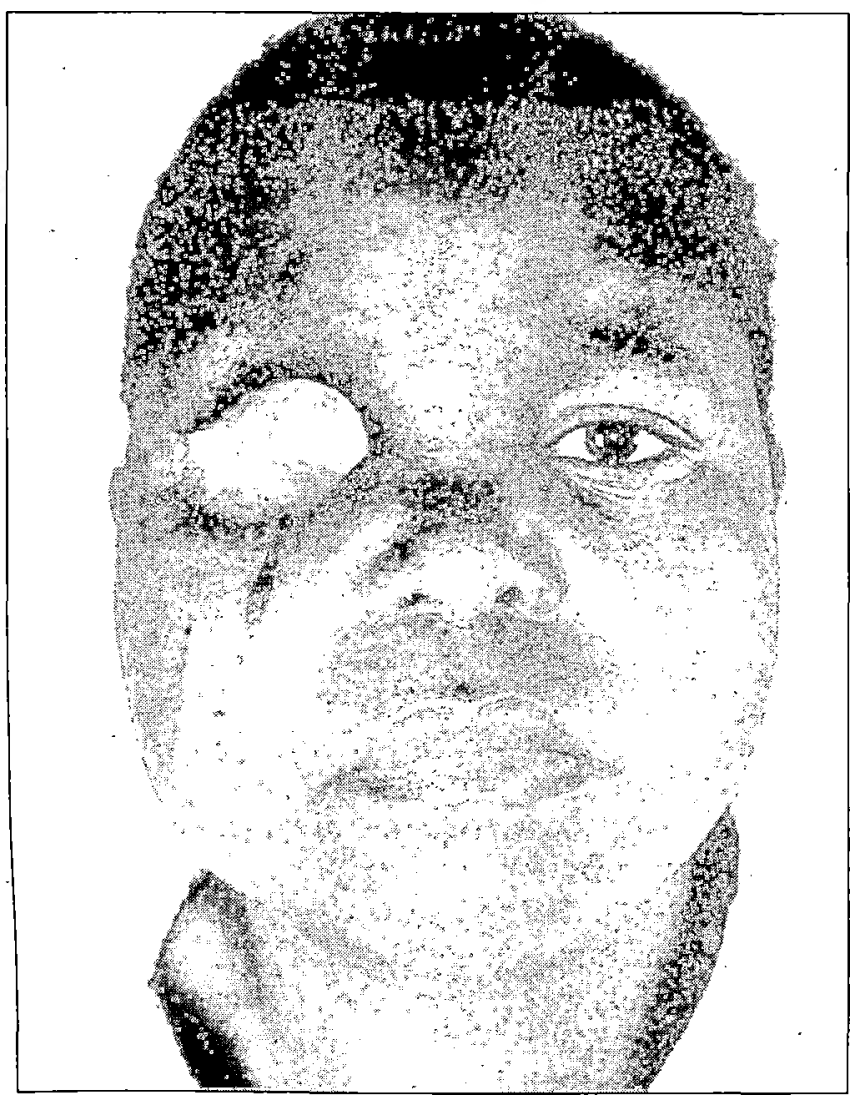

nature of the procedure. These problems contribute to the resentment of orbital exenteration by ophthalmologists and the patients.

The apparent increase in the frequency of orbital exenterations done at SKH necessitated an audit to look at the current nature of orbital tumours requiring this surgical procedure in Zimbabwe.

\section{Materials and Methods}

A hospital based retrospective study was done to look at records of all patients who had undergone orbital exenteration between 1 January and 31 December 1999 at SKH. Histology reports on orbital exenteration specimens were reviewed as well. Data on the nature of orbital disease, age, sex and eye involvement for each patient were collected. HIV status for all patients diagnosed as having SCCA of the conjunctiva was checked with double ELISA test. STATA was employed for univariate analysis of the data.

\section{Statistical analysis.}

Data was collected and univariate analysis of all variables done using STATA.

\section{Results}

A total of 23 patients had orbital exenteration done during the period under review. Figure II compares the annual cumulative frequencies of exenterations done during 1997, 1998 and 1999 at SKH respectively. It is clear that orbital exenterations done at $\mathrm{SKH}$ are on the increase.

Figure III shows a summary of the histological diagnosis of orbital tumours identified on exenteration specimens during the year 1999. Squamous cell carcinoma of the conjunctiva has been identified as the commonest tumour found in exenteration specimens at SKH during 1999. All 13 patients with a diagnosis of SCCA of the conjunctiva tested positive for HIV infection.

One male patient had CA breast secondaries in the orbit which presented years after mastectomy for a primary breast carcinoma. The patient with a basal cell carcinoma of the orbit had oculocutaneous albinism. The five unreported specimens could not be traced between theatre

Figure II: Comparison of annual cumulative frequencies of exenterations.

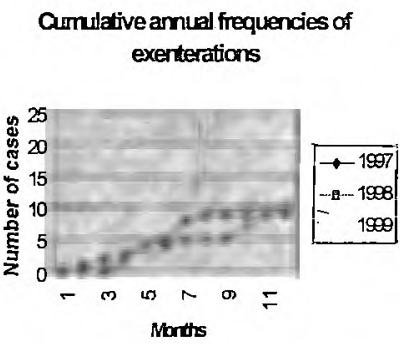


Figure III: Histological types of tumours exenterated in 1999.

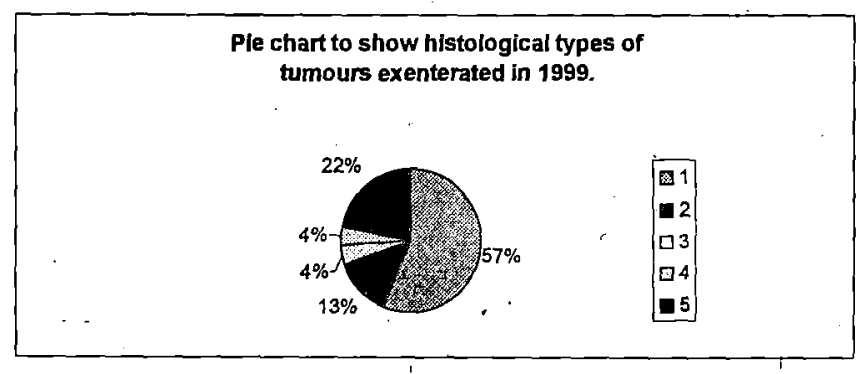

Key: 1 - Squamous cell carcinoma of conjunctiva; 2 - retinoblastoma; 3 basal cell carcinoma; 4 -breast CA secondaries to orbit; 5 - unreported.

and the histology laboratory although the histology requisition forms had been completed in theatre.

A summary of demographic data for all the 13 patients with SCCA of the conjunctiva operated in 1999 is contained in the table below.

Table I: Summary of demographic data of 1999 patients with SCCA of conjuctiva

\begin{tabular}{lll}
\hline Age iń years & Sex & Laterality \\
\hline $\begin{array}{l}\text { Mean age } 37.2 \\
\text { Interquartile range } 28,49 .\end{array}$ & Females 9 & Might eye 7 \\
\hline
\end{tabular}

There was a statistically significant difference in sex distribution of the 13 patients with SCCA of the conjunctiva who under went orbital exenteration in 1999 (male to female ratio of $1: 2.25$ at $p=0.016$ ).

\section{Discussion}

Our audit has shown that the number of orbital exenterations performed at SKH has doubled during the past year. This has been due to an increasing number of patients presenting with advanced SCCA of the conjunctiva who required orbital exenteration: The increase in number of patients suffering from SCCA of the conjunctiva is a new phenomenon which could be attributed to the AIDS pandemic. This observation has been strengthened by the positive HIV test recorded in all patients who had SCCA of the conjunctiva.

Squamous cell carcinoma of the conjunctiva is not a rare condition in Zimbabwe as some ophthalmologists from other regions suggest. ${ }^{5}$ Our audit has revealed that $56.5 \%$ of orbital exenteration specimens, whose primary site was the conjunctiva in 1999 had SCCA. In 1997 a similar observation had been made by Johnson et al in Saudi Arabia where $54.5 \%$ of orbital tumours were SCCA of the conjunctiva. ${ }^{6}$ These observations between the two population groups (black Zimbabweans and Asians) has no apparent explanation other than abundance of sunshine enjoyed by the two nations which may cause solar radiation. It is likely that the prevalence of SCCA of the conjunctiva among exenterated specimens in our setting is much higher than the figure quoted because about $20 \%$ of the specimens were not reported on, as explained above.

The high rate of orbital exenteration in the management of SCCA of the conjunctiva in Zimbabwe could be attributed to several factors, namely: late presentation of patients with conjunctival tumours, an aggressive type of tumour being seen in this region and a possible link with HIV infection. Evidence suggesting an association of solar radiation and HIV infection with SCCA of the conjunctiva has been documented. ${ }^{1,2}$ HIV tests done on all the 13 patients with SCCA were positive. Unfortunately CD4 counts were not done because of socio-economic constrains. However, the role of human papilloma virus in the aetiology of the tumour remains debatable. ${ }^{7}$

It is interesting to note that our study showed a statistically significant preference for females over males $(p=0.016)$. This observation supports the findings made by previous authors. ${ }^{8}$ The reason for this sex disparity in our audit was not clear and the small sample size could have introduced some bias in the results. A larger prospective study on SCCA of the conjunctiva in this region would enlighten us on the natural history of the disease and its outcome to various management regimes.

The mean age of 37.2 years $(I / R 28,49)$ corresponds to that of HIV sufferers in developing countries in general. This age group comprises people who are both economically and socially active in their communities. The high morbidity and mortality associated with this disease is likely to increase the burden of poverty among communities where national social security schemes are non-existent.

\section{Conclusions}

Squamous cell carcinoma of the conjunctiva is on the increase and is the commonest malignancy of the orbit necessitating. orbital exenteration in Zimbabwe today. People with persistent conjunctival growths must, therefore, consult an ophthalmologist early to facilitate detection and treatment of these tumours at an early stage. An awareness campaign focusing on the need for people with such lesions to consult eye doctors should be launched through the media.

Close follow up of all patients reported as having had malignant lesions is essential to ensure early detection of recurrences. This can be facilitated by establishing an ocular oncology clinic within the eye unit to co-ordinate the various activities.

\section{References}

1. Atenyi-Agaba C. Conjunctival squamous cell carcinoma association with HIV in Kampala, Uganda. Lancet 1995;345:695-6.

2. Waddell KM, Lewallen S, Lucas SB, Atenyi-Agaba $C$, Herrington CS, Liomba G. Carcinoma of the conjunctiva and HIV infection in Uganda and Malawi. Br J Ophthalmol 1996;80:503-8. 
3. Kestelyn PH, Stevens AM, Ndayambanje A. HIV and conjunctival malignancies. Lancet 1990;336:512.

4. Bacskulin A, Ehrardt M, Strietzel M, Pau HW, von Schwanewede $H$, Gluthoff $R$. An adjuvant afterloading brachy therapy device for use after orbital exenteration in patients with orbital malignancies. $\mathrm{Br}$ $J$ Cancer 1996;6:484-8

5. Newton R.A review of the aetiology of squamous cell carcinoma of the conjunctiva. $B r J$ Cancer $1996 ; 74: 1511-3$
6. Johnson TE; Tabbara KF, Weatherhead RG, Kersten RC, Rice C, Nasr AM. Secondary squamous cell carcinoma of the orbit. Arch Ophthalmol 1997;115:75:8.

7. Lewallen S, Shroyer KR, Keyser RB, Liomba G. Aggressive conjunctival squamous cell carcinoma in three young Africans. Arch Ophthalmol 1996;114:215-8.

8. Lewallen S, Courtright P. HIV and AIDS and the eye in developing countries. Arch Ophthalmol 1997; 115:1291-5. 


\section{(c) (1) (9)}

This work is licensed under a

Creative Commons

Attribution - NonCommercial - NoDerivs 3.0 License.

To view a copy of the license please see:

http://creativecommons.ora/licenses/bv-nc-nd/3.0/ 